\title{
PARTICIPACIÓN CIUDADANA EN LOS COMITÉS ÉTICOS INSTITUCIONALES DE INVESTIGACIÓN CLÍNICA Y ASISTENCIALES DE CATALUÑA
}

\begin{abstract}
Montse Moharra $^{1}$, Joan M. V. Pons ${ }^{1}$, Cari Almazán로 Salvador Quintana²
Resumen: Objetivo: Conocer el nivel de participación de pacientes, usuarios y ciudadanos en los comités de ética de la investigación (CEI) y los comités de ética asistencial (CEA) acreditados en Cataluña. Material y métodos: Estudio descriptivo transversal en una muestra oportunista de 30 CEI y CEA. Se administró un cuestionario dirigido a los presidentes y a los representantes de usuarios o ciudadanos (miembro lego). Para la interpretación de los resultados se analizó medias y desviación estándar. Las respuestas a las preguntas de texto abierto se analizaron mediante un análisis cualitativo del contenido. Resultados: Del total de los 30 comités de ética (CEI y CEA) contactados, 12 (40\%) aceptaron participar. En total se realizaron 15 entrevistas (9 miembros de CEI y 6 miembros de CEA): 8 presenciales, 4 telefónicamente y 3 contestadas por correo electrónico. Resultados de los CEI: del total de 7 CEI entrevistados, 2 tenían representación ciudadana en su comité. El perfil correspondía a una mujer, de profesión administrativa y a un hombre de profesión auditor contable, ambos de más de 50 años. Resultados de los CEA: de los 5 CEA que participaron, 2 declararon tener representación de la ciudanía. El perfil correspondía a una mujer, de profesión administrativa y a un hombre de profesión profesor, ambos de más de 50 años. Discusión: Actualmente hay poca representación de la ciudadanía/pacientes en los comités éticos institucionales. Constituye un tema actual de debate la necesidad de incorporar el punto de vista del ciudadano/paciente, habiendo, sin embargo, un desconocimiento sobre el perfil más idóneo y en discusión su representatividad.
\end{abstract}

Palabras clave: comités de ética, investigación, comités de ética institucionales, participación pública, participación del paciente, comité de ética clínica

\section{Participation of civilians in Catalonian clinical research and health care institutional ethical review committees}

\begin{abstract}
Aim: To know the level of participation of patients, users and civilians in scientific ethical review committees (SERC) and health care ethical review committees (HCERC) accredited in Catalonian. Materials and methods: Descriptive transversal study using an opportunistic sample of 30 SERC and HCERC. A questionnaire was filled out addressed to presidents and consumer or civilian (lay member) representatives. For the interpretation of results means and standard deviations were analyzed. Answers to open texts questions were analyzed using qualitative content analysis. Results: 12 committees (40\%) accepted to participate; 15 interviews were carried out (9 SERC members and 6 HCERC members): 8 person to person, 4 by phone and 3 by email. SERC Results: Of 7 SERC interviewed, 2 have a civilian representative in the committee. The profile was a woman of administrative profession and an auditor accountant man, both older than 50 years. HCERC Results: Of 5 HCERC participating, 2 declared having a civilian representative. The profile was a woman of administrative profession and a man teacher, both older than 50 years. Discussion: Currently, there is low civilian/patient representation in institutional ethical review committees. Today, the need to incorporate the point of view of the civilian/patient is a debatable issue, being unknown the suitable profile and their representativeness is under discussion.
\end{abstract}

Key words: ethical review committees, research, institutional ethical review committees, public participation, patient participation, health care ethical review committee

Participaçáo dos cidadãos na pesquisa clínica institucional das comissóes éticas e cuidado na Catalunha

Resumo: Objetivo: Conhecer o nível de participação de pacientes, usuários e cidadãos em comitês de ética de pesquisa (CEI) e as comissōes de ética de cuidados de saúde (CEA) acreditados na Catalunha. Material e métodos: Estudo descritivo, transversal em uma amostra oportunista de $30 \mathrm{CEI}$ e CEA. Um questionário dirigido aos presidentes e representante dos usuários ou cidadáos (membro de leigo) foi administrado. Para a interpretaçáo dos resultados foi feita uma análise que incluiu as médias e o desvio-padrão. Analisaram-se as respostas às perguntas do texto aberto com uma análise qualitativa do conteúdo. Resultados: Do total dos 30 comitês de ética (CEI e CEA) contatados, $12(40 \%)$ concordaram em participar. No total foram realizadas 15 entrevistas ( 9 membros do CEI e 6 membros da CEA). De todas as entrevistas realizadas, 8 foram presenciais, 4 foram realizadas por telefone e 3 foram respondidas por e-mail. Resultados da CEI: do total de 7 entrevistados da CEI, 2 tinham representação cidadã na sua Comissão. O perfil correspondia a uma mulher, de carreira administrativa e a um home de profissão auditor contábil, ambos com mais de 50 anos. Resultados da CEA: dos 5 CEA que participaram, 2 declararam ter representaçáo cidadá. O perfil correspondente era de uma mulher, de carreira administrativa e a um homem professor, ambos com mais de 50 anos. Discussão: Atualmente há pouca representaçáo dos cidadãos/pacientes em comitês de ética institucionais. A necessidade de incorporar o ponto de vista do cidadáo/paciente constitui um tema atual de discussão, no entanto, falta conhecimento sobre o perfil mais adequado e discussáo sua representatividade

Palavras-chave: comitês de ética, pesquisa, comitês éticos institucionais, participação pública, participação do paciente, comitê de ética clínica

\footnotetext{
${ }^{1}$ Agència de Qualitat i Avaluació Sanitàries de Catalunya (AQuAS). CIBER Epidemiología y Salud Pública. Barcelona, España Correspondencia: mmoharra@gencat.cat

${ }^{2}$ Comité de Ética de Investigación clínica con Medicamentos del Hospital Mútua de Terrassa, Barcelona España
} 


\section{Introducción}

\section{La participación ciudadana en salud}

"Participación ciudadana" es un concepto que engloba un amplio abanico de procesos y actividades que, en el contexto de la salud y de las políticas sanitarias, supone unas razones fundamentales complejas. Podríamos decir que la participación es cualquier intervención de los particulares en actividades públicas mediante el ejercicio de sus derechos civiles(1). La participación está reconocida como un derecho democrático, especialmente desde la perspectiva del usuario-consumidor de un servicio (atención sanitaria, educación) en la que el sujeto es el objeto y quien mejor puede juzgar su calidad y resultados.

Dentro de un sistema sanitario se diferencian distintos niveles de participación con temáticas decisorias diferentes: el nivel macro, de políticas, incluye elementos de financiación, cobertura, priorización, así como el encaje con las otras políticas públicas (educación vivienda, pensiones, infraestructuras, etc.); el nivel meso, de las organizaciones sanitarias, tiene en cuenta las distintas líneas de servicios que compiten entre ellas por unos recursos limitados y aspectos de accesibilidad, calidad, seguridad y equidad en la provisión; finalmente, el nivel micro comprende la relación entre profesional de la salud y persona (sana o enferma) y en el que se aboga por el abandono del modelo paternalista y la adopción de un modelo deliberativo en lo que se ha denominado "proceso de decisiones compartidas" $(2,3)$.

La participación de los pacientes en los sistemas de salud viene impulsada por la Organización Mundial de la Salud (OMS), con la Declaración de Alma-Alta en 1978 y la Carta de Ottawa de 1986(4). Más recientemente, la OMS ha centrado el tema de la participación en aspectos de seguridad del paciente y, en esta línea, está el informe publicado en 2013, en el que se plantean cuestiones sobre la toma de decisiones compartidas y la interacción entre pacientes y los sistemas de salud(5). En Europa, el Libro Blanco reitera también la importancia de la participación tanto en el plano individual como en el colectivo. En España, la ley 14/1986, que crea el Sistema Nacional de Salud (SNS), ya habla de la participación comunitaria y de los
Consejos de Salud a nivel territorial, a semejanza de los consejos de salud comunitaria del National Health Service del Reino Unido, creados en 1973. Los derechos y obligaciones de pacientes, usuarios y profesionales del SNS son regulados por la ley 41/2002, de autonomía del paciente y derechos y obligaciones en materia de información y documentación clínica.

Distintas organizaciones internacionales desarrollan actividades relacionadas con la participación de los pacientes y ciudadanos, como por ejemplo la International Alliance of Patient's Organizations (IAPO)(6), que se presenta como la voz global de los pacientes y es la promotora de la Declaration on Patient-Centred Healthcare del 2006. El European Patients' Forum(7) (EPF), organización en defensa de la salud que trabaja en el ámbito europeo con grupos de pacientes con enfermedades crónicas específicas o coaliciones nacionales de pacientes. La European Health Alliance(8) es una ONG con voluntad de liderazgo en salud pública en Europa y que trabaja para reducir las desigualdades en salud. Entre sus funciones hay el seguimiento de procesos de toma de decisiones en las instituciones de la Unión Europea. La European Patients' Academy (EUPATI)(9) es una innovadora iniciativa paneuropea en el ámbito de los fármacos, en la que participan 33 organizaciones; está dirigida por el Foro Europeo de Pacientes y se centra en la educación y la formación para aumentar la capacidad de los pacientes de comprender y contribuir a la investigación y el desarrollo de fármacos, y mejorar además la disponibilidad de información objetiva, fiable y fácil para el público.

Es importante destacar la figura de los defensores de los pacientes (patient advocates), pacientes y/o familiares activos, participativos, formados y que de forma voluntaria ayudan a otros pacientes a navegar por el sistema de salud. El patient advocate juega un papel de interlocutor válido entre el profesional y los propios pacientes. En el ámbito internacional empezó hacia 1970, con el objetivo de promover la involucración activa de los miembros de comunidades locales en la planificación y desarrollo de estudios de investigación(10). Por ejemplo, en Estados Unidos y en el caso del HIV/ SIDA, tuvieron en los años 80 un papel clave en la reclamación de cambios en las normativas que gobernaban el acceso a intervenciones que no ha- 
bían sido aprobadas por la FDA y pudieran ser accesibles para aquellos enfermos en situación más grave. Aunque la acción se inició a partir de los activistas del SIDA, las medidas políticas tuvieron también un impacto más amplio a otras enfermedades como el Alzheimer, el Parkinson o las enfermedades raras.

Por otra parte, las activistas del cáncer de mama también tuvieron en su momento un gran impacto al reclamar más recursos para la investigación y atención de las pacientes afectadas. Algunos de los potenciales beneficios de incorporar los patient advocates en la investigación están en el realce de las dimensiones sociales y morales de la investigación biomédica. Este hecho va relacionado con la idea de que la investigación no debe realizarse para satisfacer a la comunidad científica, sino para proveer beneficios en salud a la sociedad. El acto de consultar a la comunidad y a los pacientes promueve el respeto hacia las personas y asegura que sus intereses son centrales para el proyecto o estudio y no que serán tratados solo como objetos de estudio.

El Gobierno de la Generalidad de Catalunya aprobó en 2012 la creación del Consejo Consultivo de Pacientes (Consell Consultiu de Pacients), como órgano de participación y consulta entre el Departamento de Salud y las asociaciones de pacientes en el desarrollo de las políticas sanitarias, elaborar recomendaciones para la mejora de los servicios públicos de salud, así como en las actuaciones específicas para colectivos de pacientes concretos. Este Consejo en su Plan Estratégico 20122016(11) incorpora la promoción de la seguridad de la atención mediante la participación activa del paciente. Asimismo, argumenta que los pacientes son el agente principal del proceso asistencial $\mathrm{y}$ es necesario proporcionar las herramientas para favorecer la participación activa. Igualmente, los derechos y deberes de los ciudadanos y ciudadanas están recogidos en la Carta de Derechos y Deberes (Carta de drets $i$ deures)(12) de la ciudadanía en relación con la salud y la atención sanitaria, que fue aprobada por el Departament de Salut en 2001 y actualizada en 2015. En el ámbito de la participación, la carta incorpora el deber de expresar la opinión sobre el sistema sanitario y participar como agentes activos, tanto en el marco asistencial como en el de la investigación.

\section{Los comités de ética institucionales}

Hay que distinguir dos tipos de comités éticos institucionales: los comités de ética de la investigación y por otra parte los comités de ética asistencial.

\section{Los comités de ética de investigación (CEI)}

Desde el Código de Nuremberg y subsiguientes declaraciones internacionales referidas a los principios éticos de la investigación en humanos (Declaración de Helsinki, adoptada en 1964 y actualizada en el 2013, el Informe Belmont de los EEUU), a menudo como consecuencia de escándalos de prácticas inaceptables, la investigación biomédica ha estado sometida a un grado creciente de supervisión, en la que - aparte de la legislación específica que pueda existir - un elemento capital son los comités éticos de la investigación (CEI), que tienen como misión salvaguardar la protección de los derechos, seguridad y bienestar de los seres humanos que participan en proyectos de investigación, y que les puedan comportar algún riesgo físico o psicológico; además, deben dar garantía pública, evaluando la corrección metodológica, ética y legal de estos proyectos y haciendo al mismo tiempo seguimiento de su realización.

La primera regulación en España es del año 1978 (RD944/1978), con el reconocimiento de los comités de ensayos clínicos en algunos hospitales universitarios. Desde 1990 y a partir de la Ley del Medicamento 25/1990 se establece la regulación de los ensayos clínicos y se exige que éstos estén aprobados por un informe del comité ético de investigación (llamados entonces comités éticos de investigación clínica). En 1993 un Real Decreto establecía la necesaria acreditación de los CEI por la autoridad sanitaria de la comunidad autónoma. La Ley 14/2007 de Investigación Biomédica constituye el marco regulador de la investigación en ciencias de la salud y de la vida.

Con la trasposición del reglamento comunitario sobre ensayos clínicos con medicamentos, mediante el decreto 1090/2015(13), se unifica la regulación a nivel europeo y se introducen novedades importantes de procedimiento.

En Catalunya, el Decreto 406/2006 del 24 de oc- 
tubre establecía los requisitos y el procedimiento de acreditación de los comités. Su constitución en una institución que tenga entre sus finalidades la actividad de investigación biomédica tiene carácter voluntario, y está sometida al trámite de acreditación por parte de la Dirección General de Recursos Sanitarios del Departament de Salut, con carácter previo al inicio de sus actividades. Actualmente existen 35 CEI acreditados en Catalunya, aunque con muy distinto volumen de actividad. Los CEI deben de estar constituidos por un mínimo de diez miembros, y con los siguientes perfiles: tres médicos, uno de los cuales ha de ser especialista en farmacología clínica; un farmacéutico o farmacéutica especialista en farmacia hospitalaria; un farmacéutico o farmacéutica de atención primaria; un diplomado o diplomada en enfermería; una persona adscrita a una unidad de atención al usuario de uno de los centros, servicios o establecimientos sanitarios que forman parte del ámbito de actuación acreditado por el comité; dos personas ajenas a las profesiones sanitarias, una de las cuales ha de ser licenciada en Derecho, especialista en la materia; una persona miembro del comité de ética asistencial y una de la comisión de investigación de la institución donde se constituye el comité.

Como mínimo, una de estas personas no ha de estar vinculada laboralmente con la institución donde se constituya ni con los centros o instituciones del ámbito de actuación acreditado del comité.

\section{Los comités asistenciales de ética (CEA)}

En la práctica médica ha sido constante la presencia de dilemas éticos. Es, sin embargo, en la segunda mitad del siglo XX, con el desarrollo científico y tecnológico (máquinas de soporte vital, trasplante, etc.), que su presencia se hace más acuciante. Sirvan de ejemplo la comisiones para la priorización de pacientes en el acceso a las primeras máquinas de hemodiálisis en los años 50 y 60 o el Comité de la Facultad de Medicina de Harvard que, en 1968, estableció criterios para determinar la muerte cerebral y posibilitar, entre otras cosas, órganos para trasplante.

Los CEA son unos órganos de apoyo consultivo, no vinculante (excepto en el caso de los trasplantes de órganos de donante vivo), con visión plural y de composición multidisciplinar, que tienen como objetivo ayudar a los profesionales y usuarios de una institución sanitaria ante dilemas éticos que se pueden plantear, promoviendo a la vez la formación en éste ámbito de la bioética y elaboración de recomendaciones si es el caso. Los informes que el CEA elabora contiene información que tan solo pretende ofrecer ayuda al profesional para adoptar una decisión con fundamentos éticos(14).

En España, la primera regulación de los CEA se encuentra en la Circular del INSALUD de 30 de marzo de 1995, de la Dirección General del Instituto Nacional de Salud de acreditación de Comités Asistenciales de Ética. Dentro del ámbito autonómico fueron territorios pioneros Cataluña (1993) y País Vasco (1995). Merece un reconocimiento particular la figura del doctor Francesc Abel, médico y teólogo, que fue el primero en España en crear una institución dedicada a la investigación y formación en bioética, inspirada en el Kennedy Institute of Ethics de Washington, y de impulsar el primer CEA en España en 1976, en el Hospital pediátrico de San Juan de Dios de Barcelona.

Los CEA deben de estar constituidos por un mínimo de siete miembros, uno de los cuales debe ejercer la presidencia y con los siguientes perfiles: médicos, diplomado o diplomado en enfermería, un profesional de la Dirección asistencial del centro, una persona ajena a la institución con interés acreditado en el campo de la ética.

Debe formar parte también del CEA un miembro de cada una de las instancias siguientes, en el caso de que exista en la institución: un profesional de la unidad de la atención al usuario, un miembro del comité ética de investigación clínica, un miembro de la comisión de calidad asistencia, un profesional de la Asesoría Jurídica o Servicio de Medicina Legal y un representante del sector sanitario o de la región sanitaria del Servei Català de la Salut, cuando se trate del comité de referencia de uno de estos órganos.

Actualmente, aun existiendo una misión bien definida para los CEI y CEA, y un marco legislativo sobre su composición y funciones, su actuación está preferentemente entre investigadores y profesionales de la salud, siendo órganos desconocidos por la mayoría de pacientes, usuarios y ciudadanos. 


\section{Objetivo}

El objetivo de este trabajo es conocer el nivel de participación de pacientes, usuarios o ciudadanos en los CEI y CEA actualmente acreditados en Cataluña. La hipótesis del estudio es que actualmente la presencia y la participación de los ciudadanos (enfermos o sanos), o de representantes de la ciudadanía en dichos comités éticos, es testimonial o de escaso impacto. Por tanto, un mayor grado de participación de los ciudadanos en los comités éticos podría aportar un punto de vista más claro sobre las necesidades, preferencias y valores de las personas incluidas en los estudios clínicos, y en aquellos dilemas asistenciales que puedan afectarlos.

\section{Material y métodos}

Estudio transversal mediante entrevista presencial o telefónica a través de un cuestionario semiestructurado, dirigida a los presidentes y al representante de los usuarios (miembro lego). La población de estudio fue una selección oportunista de 15 CEI y CEA de la lista de centros acreditados(15). Se intentó que la muestra de los comités fuera representativa en cuanto a distribución territorial y tamaño de los comités. Se contactó vía email con el presidente de los comités seleccionados. El cuestionario incluía preguntas que ayudaban a describir los comités de ética, pero también, y de forma más específica, preguntas que permitían conocer la implicación y participación de pacientes, usuarios y ciudadanos en los CEI y CEA.

Se enviaron un total de 30 correos electrónicos a 15 CEI y 15 CEA solicitando la entrevista personal o la administración vía telefónica o por correo electrónico. El periodo de estudio desde que se enviaron los correos electrónicos hasta la realización de la última entrevista comprendió los meses de abril a septiembre de 2017. Durante este periodo se efectuaron tres recordatorios a los comités en los cuales no se había obtenido respuesta o habían delegado la respuesta a otro miembro del comité de ética. Para la interpretación de los resultados se hizo un análisis que incluyó medias y desviación estándar de modo de caracterizar a los comités entrevistados. Las respuestas a las preguntas de texto abierto se analizaron mediante un análisis cualitativo del contenido.

\section{Resultados}

Del total de los 30 comités de ética (CEI y CEA) contactados, $12(40 \%)$ aceptaron participar. En total se realizaron 15 entrevistas (9 miembros de CEI y 6 miembros de CEA). Del total de entrevistas, 8 fueron presenciales, 4 se realizaron telefónicamente y 3 fueron contestadas por correo electrónico.

\section{Resultados de las entrevistas a los miembros de los CEI}

Los CEI tenían 16,8 $(\mathrm{DE} \pm 3)$ miembros de media. La mayor parte de CEI entrevistados, 71,4\%, no cuenta con representación ciudadana o de pacientes. El representante no sanitario del comité es quien suele tener el rol en el comité de revisar los consentimientos informados y valorar el riesgo/ beneficio desde el punto de vista del ciudadano, a la vez también vela por el cumplimiento de los derechos y deberes en relación con la salud. En los 2 CEI que afirmaron que tenían representación ciudadana en su comité, el perfil de estas personas correspondía a una mujer, de profesión administrativa, y a un hombre de profesión auditor contable, ambos en un rango de edad de más de 50 años. La tabla 1 muestra el perfil de los CEI entrevistados.

Los miembros entrevistados destacaron que el hecho de no tener representación ciudadana es un tema actual de debate, pero hay dudas sobre el perfil que deberían tener estos representantes. Podrían ser pacientes provenientes de alguna asociación, pero la imparcialidad sería difícil y es importante mantener la transparencia y la ecuanimidad en el comité. Cabe destacar que gran parte de los miembros entrevistados reportan el perfil del filósofo como un miembro importante dentro del comité, por su contribución a la reflexión y deliberación.

En cuanto a la tarea que desempeñan en el comité, ambas personas declararon que mayoritariamente su tarea se focaliza en asegurar que el texto que se presenta en los consentimientos informados es redactado de forma clara y entendedora. Una de las personas entrevistadas se integró como representante de la ciudadanía en el 2007 y explicó que, cuando empezó, los textos del consentimiento informado eran inteligibles para los pacientes. 
Tabla 1. Perfil de CEI entrevistados*

\begin{tabular}{|c|c|c|c|c|}
\hline & & \multicolumn{3}{|c|}{ Ámbito de actuación del CEI } \\
\hline CEI & $\begin{array}{c}\text { Número de proyectos } \\
\text { presentados y aprobados }\end{array}$ & $\begin{array}{c}\text { Número de centros } \\
\text { hospitalarios }\end{array}$ & $\begin{array}{c}\text { Número de centros } \\
\text { extrahospitalarios }\end{array}$ & $\begin{array}{c}\text { Número de } \\
\text { centros no } \\
\text { sanitarios }\end{array}$ \\
\hline CEI 1 $^{\text {a }}$ & 156 & 32 & 48 & 16 \\
\hline CEI 2 & 224 & 5 & 1 & 0 \\
\hline CEI 3 & 75 & 3 & 10 & 1 \\
\hline CEI 4 & 104 & 3 & 10 & 6 \\
\hline CEI 5 & 63 & 10 & 10 & 2 \\
\hline CEI 6 & 153 & 9 & 43 & 4 \\
\hline CEI 7 & 470 & 2016 & 10 & 4 \\
\hline
\end{tabular}

*Información extraída de la memoria de actividades 2016 del CEI; ªFusión de dos CEI.

Los dos representantes manifestaron que asistían regularmente a las sesiones del CEI y que creían que sus opiniones y aportaciones se tenían siempre en cuenta cuando era el momento de aportar su visión. Hasta recientemente, cualquier investigación clínica y documentación acompañante debía ser aprobada por el CEI local donde se llevaba a cabo la investigación, con lo cual su rol era muy importante, sobre todo en relación a los fármacos y a las pruebas que se hacía a las personas. El cambio legislativo que ha supuesto la trasposición del reglamento de la Unión Europea, que establece un solo CEI que apruebe el protocolo de estudio, relega a los otros CEI de las instituciones participantes en el estudio.

Con relación a la formación recibida o a la necesidad de tener una cierta formación para formar parte del comité, ambos representantes declararon que no es imprescindible recibir o tener una formación en bioética específica para formar parte del comité. No obstante, internamente se ofrece formación cuando hay la opción de asistir a sesiones o dependiendo también de los recursos disponibles, pero no es una actividad obligada como miembro activo del comité.

\section{Resultados de las entrevistas a los miembros de los CEA}

Los CEA tenían 13,8 (DE \pm 4$)$ miembros de media. De los 5 CEA que participaron, dos declararon tener representantes de la ciudanía. En cuanto al rol que desempeñan, en general son quienes aportan la visión de los pacientes y los usuarios, participando en las discusiones de los casos y en la deliberación como cualquier otro miembro del comité. En cuanto al perfil del representante de la ciudadanía, correspondía a una mujer, de profesión administrativa y a un hombre de profesión profesor, ambos en un rango de edad de más de 50 años. Los CEA entrevistados que declararon no tener representación de la ciudadanía, $60 \%$, reportaron durante la entrevista que el miembro ajeno a la institución, con interés acreditado en el campo de la ética, recae en un filósofo y es quién representa de alguna manera a los ciudadanos, ya que no trabaja en la organización ni es tampoco profesional de la salud.

Los temas que mayoritariamente se tratan en el CEA están relacionados con decisiones al final de la vida o temas de trastornos mentales, como puede ser la incapacitación de la persona o en otros casos donde la autonomía pueda estar muy limitada. En cuanto a la formación, en general no se solicita como requisito para entrar en el CEA. Se pide a los miembros que asistan a las sesiones y, en este caso, se valora la formación en filosofía, la forma de razonar y la manera como pueden aportar sus valoraciones. Algunos miembros del CEA tienen formación recibida antes de entrar en el CEA y, en la mayoría de los casos, ésta se ha hecho previamente a la participación en el comité, pero no es un criterio de selección. Se valoran otros criterios, como por ejemplo decisiones sobre valores y, por tanto, esto no implica necesariamente formación. 
En relación a la comunicación con el paciente o familiares, normalmente es el profesional quien representa el caso delante del CEA y es él quien establece la comunicación con la familia o paciente. En general, la familia no tiene la costumbre de pedir asesoramiento al CEA. No obstante, siempre se atienden las demandas que puedan venir de la familia o del paciente. Si este es el caso, siempre se da respuesta a la familia o paciente que hace la demanda, y se valida con el profesional sanitario sobre la pregunta o consulta. Se intenta también conocer el motivo por el cual el profesional no ha derivado la consulta al CEA.

\section{Discusión}

Los resultados del estudio indican que actualmente hay poca representación de la ciudadanía/ pacientes en los comités éticos de investigación y de ética asistencial. Del total de los 12 comités entrevistados, solo un tercio $(\mathrm{N}=4)$ declaró tener representación de pacientes o de la ciudadanía. No obstante, la gran mayoría de los miembros entrevistados estuvieron de acuerdo en la necesidad de incorporar el punto de vista del ciudadano/ paciente, porque puede representar más fielmente a la sociedad (cultura, tradición, valores), ya que se trata de miembros independientes, sin intereses hacia la institución, y porque su aportación es más desde el punto de vista del paciente. Sin embargo, hay desconocimiento sobre el perfil más idóneo, como también sigue en cuestión el grado de representatividad.

La presencia de ciudadanos o pacientes como miembros de los comités institucionales (éticos de investigación y éticos asistenciales) es un tema de debate actual. Podrían ser personas provenientes de alguna asociación de pacientes o de vecinos, pero sin menoscabo de la ecuanimidad del comité. Cabe destacar que gran parte de los miembros entrevistados (CEI y CEA) reportan el perfil del filósofo como un miembro importante dentro del comité, ya que se aproxima a la visión y/o aportación ciudadana, por su perfil de ayuda a la reflexión y deliberación en la mayoría de casos que se exponen. La importancia de incluir el perfil de la ciudadanía en los comités de ética asistencial (CEA) ha sido un tema discutido en otros estudios así como también la reflexión del perfil más apropiado. Es importante remarcar que, finalmente, quienes mejor se pueden beneficiar, especialmente en los CEA, son los propios ciudadanos y, por tanto, es relevante reflexionar y revisar la composición actual de miembros legos en los comités, así como también el perfil que deben tener. La American Society for Bioethics and Humanities propone un total de 26 competencias que los miembros de los CEA deben reunir clasificadas en 3 áreas: a) habilidades de contenido o juicios éticos, procedimentales y personales; b) conocimientos relevantes para abordar el caso conflictivo, y c) actitudes o virtudes. No se especifican, sin embargo, las características más específicas que deben tener en cuanto a la aportación y visión ciudadana esperada.

Los resultados de las entrevistas realizadas a los CEI y CEA en Cataluña muestran en general homogeneidad en cuanto al número y perfil de los miembros de los comités, puesto que aplican el marco de acreditación legislativo de actuación vigente, lo cual es consistente con el resultado de otros estudios sobre comités de ética. El perfil del filósofo es un miembro que suelen incorporan ambos tipos de comités, como miembro representante externo (no vinculado a la institución ni profesional de la salud). En el aspecto normativo, es importante destacar el Real Decreto 1090/2015, que tendrá validez completa en 2018 y que propicia un acercamiento de la investigación clínica a la sociedad, aumentando la participación de los pacientes en las decisiones e integrándolos de forma obligatoria en los comités de ética de la investigación.

La revisión del consentimiento informado sigue siendo una de las principales tareas que los representantes de la ciudadanía o miembros no sanitarios llevan a cabo en los CEI, asegurando que se entiende de forma clara y desarrollar la parte más humana y cotidiana de la persona que se enfrenta ante un estudio de investigación. Cabe destacar que el consentimiento informado sigue siendo aún un handicap para los investigadores que presentan estudios en los CEI, en cuanto a la metodología del estudio y a la hoja de información que se completa con el consentimiento informado(16). En cambio, en el caso de los CEA, el representante de la ciudadanía/pacientes participa en las discusiones de los casos y en la deliberación, aportando su punto de vista como cualquier otro miembro. La importancia de participar en las discusiones de los casos por parte de los familiares ha sido destacada 
en otros estudios, que remarcaban el impacto que había tenido la presencia de los familiares durante la deliberación de los casos al ayudar a entender la decisión final tomada. Los participantes tuvieron la percepción que habían sido escuchados y que habían aportado información vital a la discusión, en el sentido que se aclaraban los malentendidos o confusiones(17). La presencia de los familiares en la discusión de los casos permite hacer aflorar el interés y preferencia del paciente y, en el caso del CEA, modificar las percepciones primando la calidad de vida, como ha sido referido en los estudios de suspensión de la diálisis renal(18).

En cuanto a la formación que se imparte en los comités, cuya necesidad han recogido algunos trabajos(19), es en general de buen nivel en los comités (CEI y CEA) que han participado y en consonancia con otros estudios publicados(20-22). Es escasa, sin embargo, la formación continuada.

Por lo que hace referencia, en los CEA, al circuito de comunicación que se establece entre el paciente o la familia y el propio comité, es el profesional quien generalmente presenta el caso. No obstante, se atienden siempre las preguntas o dudas que puedan venir por parte de la familia, aunque no suele ser el proceso habitual. El hecho de que la propia persona afectada no asista a la sesión de deliberación del CEA ha sido remarcado en otros estudios como un factor relevante a tener en cuenta, puesto que no se expone una situación de igualdad en cuanto a la representatividad de los miembros CEA. Sería necesario plantear la ampliación de la representación de los ciudadanos, para evitar cualquier sesgo informativo en relación a su situación.

Cabe destacar algunas limitaciones del estudio. En primer lugar, la tasa de respuesta del $40 \%$ se puede considerar baja, a pesar de los esfuerzos y recordatorios enviados por correo electrónico. A menudo el correo electrónico era un buzón general del comité y, además, en algunos casos, las direcciones no eran correctas o no estaban actualizadas y, consecuentemente, el proceso para obtener la dirección correcta fue laborioso y en algunos casos imposible. Sin embargo, el hecho de que la entrevista fuera en la mayoría de los casos (75\%) realizada vía presencial o telefónica añadió mucha más información cualitativa complementaria y de gran ayuda en el análisis de los resultados.
El presente estudio evidencia la necesidad de desarrollar un marco de participación ciudadana (personas sanas o enfermas) en los comités de ética institucionales, sean CEI o CEA, el cual debería definir la formación, así como el perfil y funciones de dichos miembros. Su papel puede ser relevante, al poder aportar, de manera más fidedigna, los valores, preferencias y necesidades de las personas incluidas en los estudios clínicos o en aquellos retos éticos asistenciales que se presenten.

\section{Conclusiones}

Actualmente hay poca representación de la ciudadanía/pacientes en los comités éticos de investigación y de ética asistencial. La revisión del consentimiento informado sigue siendo una de las principales tareas que los representantes de la ciudadanía o profesionales no sanitarios llevan a cabo en los CEI. La importancia de incorporar la visión del ciudadano/paciente en los comités, así como también del perfil apropiado que deberían tener es un tema de debate y de reflexión. Cabe destacar que el perfil del filósofo es un miembro que suelen incorporar ambos tipos de comités como representante externo (no vinculado a la institución ni profesional de la salud).

Falta desarrollar un marco de cómo debe llevarse a cabo la participación ciudadana (personas sanas o enfermas) para que se realice de forma homogénea en los comités de ética institucionales, sean CEI o CEA. De esta manera, se podría definir la formación, así como el perfil y funciones de dichos miembros, cuyo papel puede ser relevante al poder aportar, de manera más fidedigna, los valores, preferencias y necesidades de las personas incluidas en los estudios clínicos o en aquellos retos éticos asistenciales que se presenten.

\section{Agradecimientos}

A Pau Ferrer, por su ayuda y colaboración de consulta y soporte en este trabajo de investigación. También a los documentalistas de la biblioteca del Instituto Bioética Borja por su colaboración y ayuda en la bibliografía, así como también a los miembros de los comités entrevistados por su tiempo y dedicación. A Toni Parada, documentalista, por la revisión y supervisión en el diseño de la búsqueda bibliográfica. 


\section{Referencias}

1. Segura A. La participación ciudadana, la sanidad y la salud. Ges Clin Sanit. 2010; 12(2): 55-62.

2. Millaret M, Pons JMV. Participació de ciutadans i pacients en les politiques de salut: conceptualització i experiències internacionals. Barcelona: Agència de Qualitat i Avaluació Sanitàries de Catalunya. Departament de Salut. Generalitat de Catalunya; 2015.

3. Conklin A, Slote Morris Z, Nolte E. Involving the public in healthcare policy. An update of the research evidence and proposed evaluation framework [Internet]. Cambridge (United Kingdom): RAND Europe; 2010 [consultat 20 oct 2017]. Disponible en: www.rand.org/content/dam/rand/pubs/technical_reports/2010/RAND_TR850.pdf

4. World Health Organization. Carta d'Ottawa; 1986. Disponible en: www.euro.who.int

5. World Health Organization. Exploring patient participation in reducing health-care-related safety risk. WHO; 2013.

6. International Alliance of Patients' Organizations. Declaration on Patient-Centred Healthcare. London (United Kingdom): IAPO; 2006 [consultat 20 oct 2017]. Disponible en: www.iapo.org.uk/sites/default/files/files/IAPO_declaration_ENG_2016.pdf

7. European Patients Forum (EPF). [Internet]. Brussels (Belgium): EPF; 2017 [consultat 20 oct 2017]. Disponible en: www.eu-patient.eu

8. European Health Alliance (EPHA). [Internet]. Brussels (Belgium): EPHA; 2017 [consultat 20 oct 2017]. Disponible en: https://epha.org

9. European Patients' Academy (EUPATI). [Internet]. Brussels (Belgium): EUPATI; 2017 [consultat 20 oct 2017]. Disponible a: www.eupati.eu

10. Dresser R. When Science offers salvation. Patient Advocacy and Research Ethics. Oxford (United Kingdom): Oxford University Press; 2001.

11. Consell Consultiu de Pacients de Catalunya. Pla estratègic de la participació dels pacients en el sistema sanitari públic de Catalunya 2013-2016 [Internet]. Barcelona: Direcció General d'Ordenació i Regulació Sanitàries. Departament de Salut. Generalitat de Catalunya; 2014 [consultat 20 oct 2017]. Disponible en: http://salutweb.gencat.cat/web/.content/ home/ambits_tematics/per_perfils/Consell_pacients/documents/arxius/Pla-estrategic-CCPC_revisatMarc15.pdf

12. Generalitat de Catalunya. Carta de Drets i Deures de la ciutadania en relació amb la salut i l'atenció sanitària [Internet]. Barcelona: CatSalut. Departament de Salut. Generalitat de Catalunya; 2015. [consultat 20 oct 2017]. Disponible en: http://canalsalut.gencat.cat/web/.content/home_canal_salut/ciutadania/drets_i_deures/destacats/carta-drets-deures. pdf

13. Real Decreto 1090/2015, de 4 de diciembre, por el que se regulan los ensayos clínicos con medicamentos, los Comités de Ética de la Investigación con medicamentos y el Registro Español de Estudios Clínicos. Boletín Oficial del Estado, BOE, 307, 24/12/2015.

14. Gascón M. Los Comités Asistenciales de Ética (CAE). Un año de funcionamiento del CAE del Área de Salud de Albacete. Rev Clin Med Fam. [Internet] 2010; 3(2): 110-113. [consultat 20 oct 2017]. Disponible en: http://scielo.isciii.es/ pdf/albacete/v3n2/especial1.pdf

15. Generalitat de Catalunya. Comitès d'ètica d'investigació clínica (CEIC) acreditats a Catalunya. A: Canal Salut [Internet]. Barcelona, Departament de Salut. Generalitat de Catalunya; 2011 [consultat 20 oct 2017]. Disponible a: http:// canalsalut.gencat.cat/ca/professionals/consells_i_comissions/comites_etics_dinvestigacio_clinica_ceic_acreditats/comites_etica_dinvestigacio_clinica_acreditats_a_catalunya/

16. Adams P, Wongwit W, Pengsaa K, Khusmith S, Fungladda W, Chaiyaphan W, et al. Ethical issues in research involving minority populations: the process and outcomes of protocol review by the Ethics Committee of the faculty of the Tropical Medicine, Mahidol University, Thailand. BMC Med Ethics. 2013; 14: 33.

17. Forde R, Linja T. It scares me to know that we might not have been there!: a qualitative study into the experiences of parents of seriously ill children participating in ethical case discussions. BMJ Med Ethics 2015; 16: 40.

18. Maurizi J, Cartier JC, Calvino-Gunther S, Carron PL, Baro P, Palacin P, Vialtel P. Dialysis Withdrawal: Impact and Evaluation of a Multidisciplinary Deliberation Within an Ethics Committee as a Shared-Decision-Making Model. Therapeutic Apheresis and Dyalisis 2015; 19(4): 385-392.

19. Ramos S, Morlans M. Legos en el comité de ética: una reflexión desde la ética dialógica. Revista de Bioética y Derecho 2011; (21): 33-39. [consultat 20 oct 2017]. Disponible en: http://revistes.ub.edu/index.php/RBD/article/view/7740.

20. Generalitat de Catalunya. Avaluació dels comitès d'ètica assistencial a Catalunya, 2014. Unitat de Bioètica, Departament de Salut, Generalitat de Catalunya. Julio 12014.

21. Villén N, Redondo S, Font R, Quintana S. Comités éticos: perfil de los miembros no sanitarios de los comités éticos de investigación clínica y comités de ética asistencial de Catalunya. Med Clin. 2012; 138(7): 296-299. 
Participación ciudadana en los comités éticos institucionales - Montse Moharra et al.

22. Ribas Ribas S. Estudio observacional sobre los comités de ética asistencial en Catalunya: el estudio CEA-CAT (1). Estructura y funcionamiento. Med Clin (Barc). 2006; 126(2): 60-66.

Recibido: 30 de marzo de 2018

Aceptado: 8 de junio de 2018 